Les ANNALES Les Annales de droit

DE DROIT

13 | 2019

Varia

\title{
Les règles prudentielles et les immobilisations incorporelles dans l'UEMOA
}

Prudential rules and intangible assets within WAEMU

\section{Richard Alemdjrodo}

\section{OpenEdition}

Journals

Édition électronique

URL : http://journals.openedition.org/add/1350

DOI : $10.4000 /$ add. 1350

ISSN : 2606-1988

Éditeur

Presses universitaires de Rouen et du Havre

Édition imprimée

Date de publication : 1 mai 2019

Pagination : 09-28

ISBN : 979-10-240-1293-3

ISSN : 1955-0855

Référence électronique

Richard Alemdjrodo, "Les règles prudentielles et les immobilisations incorporelles dans I'UEMOA », Les Annales de droit [En ligne], 13 | 2019, mis en ligne le 09 décembre 2019, consulté le 24 janvier 2021. URL : http://journals.openedition.org/add/1350 ; DOI : https://doi.org/10.4000/add.1350 


\section{Les règles prudentielles et les immobilisations incorporelles dans I'UEMOA}

Richard AlemDJRODo

"Si le climat était une banque, les pays riches l'auraient déjà sauvé», dixit Hugo Chávez ${ }^{1}$. L'histoire des règles prudentielles de Bâle semble lui donner raison. Elle commence avec la crise économique de 2008. Le but de ces règles est d'obliger les banques à une stricte couverture en fonds propres de leurs risques. La crise financière n'a pas seulement eu des effets négatifs sur l'exportation des pays africains ${ }^{2}$, elle a également permis aux systèmes bancaires de la zone CFA (Communauté financière africaine) - Union économique et monétaire Ouest-Africaine (UEMOA) et Communauté économique et monétaire de l'Afrique centrale (CEMAC) - de comprendre que les déséquilibres graves dans une union monétaire résultent d'arrangements qui ne sont pas conçus pour résister à une variété de chocs $^{3}$, et à adhérer aux règles prudentielles de Bâle ${ }^{4}$. L'UEMOA, notamment, par le biais de sa banque centrale, la

1. Discours prononcé par feu le président du Venezuela, Hugo Chávez, au sommet climatique des Nations Unies à Copenhague (COP15) le 16 décembre 2009 : https://mrmondialisation.org/si-le-climat-etait-une-banque-il-serait-deja-sauve/.

2. Uchenna Efobi, Evans Osabuohien, «Trade Outcomes in Africa’s Regional Economic Communities and Institutional Quality: Some Policy Prescriptions », Petroleum-Gas University of Ploiesti Bulletin, vol. 63, $\mathrm{n}^{\mathrm{o}}$ 4, 2011, p. 19-32.

3. Simplice Agonsu, «New Empirics of Monetary Policy Dynamics: Evidence from the CFA Franc Zones », African Journal of Economics and Management Studies, vol. 7 , $\mathrm{n}^{\mathrm{o}} 2$, 2016, p. 164-204.

4. Il faut cependant préciser que l'insuffisance des fonds propres des banques a été un problème majeur pour les banques des pays développés touchés, entre autres, par la crise des subprimes. Les pays émergents n'ont été touchés que plus tard. Quant aux pays les moins développés (catégorie dans laquelle on peut ranger la quasi-totalité des pays de l'UEMOA), ils n'ont pas vraiment eu à souffrir du problème de l'insuffisance des fonds propres bancaires. À ce propos, voir Marie-Françoise Delaitte, « Les fonds propres bancaires au cœur de la crise financière ", Mondes en développement, $\mathrm{n}^{\circ}{ }_{160}$, 2012, p. 117-132. 
$\mathrm{BCEAO}^{5}$, avait déjà pris un certain nombre de mesures ${ }^{6}$. Il convient de relever à ce stade la situation particulière de l'UEMOA, une communauté économique et monétaire de huit États qui a ses règles comptables ${ }^{7}$, alors que dans le même temps, ses huit États sont assujettis aux règles de constitution des sociétés commerciales de l'Organisation pour l'harmonisation en Afrique du droit des affaires (OHADA) qui comptent 17 États et qui a également son propre droit comptable ${ }^{8}$.

Parmi les éléments à prendre en considération par l'UEMOA dans les règles prudentielles se trouvent les immobilisations. Pendant longtemps, il n'a pas été facile de définir les immobilisations incorporelles, le droit comptable et le droit fiscal refusant de s'y frotter totalement. En France, l'expression apparaissait déjà dans le plan comptable général (PCG)

5. Les règles prudentielles ne sont pas une nouveauté au sein de la banque communautaire. Le guide banquier de l'UMOA (Union Monétaire Ouest-Africaine remplacée plus tard par l'UEMOA) les a prévues dans son document intitulé « Actes courants de la vie d'un établissement de crédit ». Le dispositif prudentiel complète la loi bancaire. Il est arrêté par le Conseil des ministres de l'UMOA sur proposition de la BCEAO, en application de l'article 44 de ladite loi. Il consiste en une série de dispositions organisées autour de trois thèmes : les conditions d'exercice de la profession (capital minimum et sa représentation, réserve spéciale, réglementations comptables) ; la réglementation d'opérations spécifiques (participations, immobilisations, prêts aux principaux actionnaires, aux dirigeants et au personnel); les normes de gestion (couverture des risques par les fonds propres effectifs, couverture des emplois à moyen et long termes par des ressources stables, division des risques, règles de liquidité, structure de portefeuille). Voir Guide du banquier de l'UMOA, p. 22.

6. Voir Boubacar Naby Camara, "Réglementation prudentielle et risque bancaire: incidence de la structure et du niveau du capital réglementaire ", thèse soutenue publiquement le 7 décembre 2010 pour obtenir le grade de docteur de l'université de Limoges en sciences économiques, http://www.theses.fr/2010LIMO1002.

7. Voir Nadédjo Bigou-Laré, "Le SYSCOA, vers des normes comptables uniformes dans l'UEMOA ? Une analyse de la pratique togolaise », Comptabilité, contrôle, audit, vol. 10, $\mathrm{n}^{\mathrm{o}} 1$, 2004, p. 63-78.

8. Malgré l'adoption de l'Acte uniforme portant organisation et harmonisation des comptabilités des entreprises, le Conseil des ministres de l'UEMOA a décidé, le 20 septembre 2001, à Dakar, de maintenir le SYSCOA en application dans cet espace depuis le $1^{\mathrm{er}}$ janvier 1998 , tout en procédant à des aménagements concernant le dispositif juridique et technique, afin de le mettre en harmonie avec l'Acte uniforme. Ainsi, le SYSCOA révisé et harmonisé a donné naissance au SYSCOHADA pour renforcer l'OHADA qui regroupe non seulement les États membres de l'UEMOA, mais aussi ceux de la CEMAC et des pays indépendants. Le SYSCOHADA est donc un référentiel comptable conçu pour l'espace concerné et qui intègre les caractéristiques fondamentales qui ont prévalu dans l'élaboration des référentiels comptables jusqu'alors utilisés dans les États parties. Dans le cadre de cet article, il sera fait essentiellement appel au SYSCOHADA. 
de $1957^{9}$, dans une sous-rubrique du compte d'immobilisations ${ }^{10}$ qui regroupait le fonds de commerce et droit au bail, les brevets, licences, marques, procédés, modèles, dessins, concessions, ainsi que les comptes de dépréciation (amortissement et provision) s'y rapportant. Les éléments inscrits dans ces comptes l'étaient pour leur valeur d'acquisition dans les mêmes conditions que les immobilisations corporelles. Un arrêt de la Cour administrative d'appel de Marseille a rendu une décision pour pallier l'absence d'une définition de la loi fiscale. Elle a ainsi décidé qu'une immobilisation doit, pour être qualifiée d'incorporelle, outre le fait d'être dépourvue de substance physique, constituer une source régulière de profit pour l'entreprise et être dotée d'une pérennité suffisante, être un bien identifiable distinctement de l'activité de l'entreprise et cessible, ou bien doit avoir pour origine une protection juridique résultant d'un droit légal ou contractuel, ces deux critères étant alternatifs ${ }^{11}$. Le droit comptable OHADA la définit comme étant des immobilisations immatérielles et tous les autres éléments susceptibles de générer des avantages futurs ${ }^{12}$. Elles ont la nature de biens acquis ou créés par l'entreprise, non pour être vendus ou transformés, mais pour être utilisés de manière durable, directement ou indirectement, pour la réalisation des opérations professionnelles ou non. La zone UEMOA qui a hérité du droit comptable français, avant de disposer de son propre principe comptable, considère comme immobilisations incorporelles les frais de recherche et développement ${ }^{13}$, les brevets, les

9. Ce plan est lui-même dérivé du plan établi durant la seconde guerre mondiale et révisé en 1947. Voir Jean-Luc Rossignol, «La problématique comptable et fiscale des éléments incorporels ( ${ }^{\text {re }}$ partie) $)$, Petites Affiches, ${ }^{\circ}{ }^{140}$, 2011, p. 4-15.

10. Elles se mêlent ainsi aux autres éléments d'actifs corporels.

11. CAA Marseille, $4^{\mathrm{e}}$ chambre, 17 décembre 2013, $\mathrm{n}^{\mathrm{o}} 12 \mathrm{MA0} 3378$, Inédit au recueil Lebon.

12. Voir Annexes, chap. 2 (Contenu et fonctionnement des comptes), section 2, classe 2 (comptes d'actifs immobilisés), compte 21 (immobilisations corporelles), http://www.ohada.com/actes-uniformes/693/734/compte-21-immobilisationscorporelles.html.

13. Cette traduction comptable se fonde sur la distinction entre frais de recherche, frais de recherche appliquée et frais de recherche et de développement. Alors que les frais de recherche fondamentale ne peuvent être portés à l'actif en raison de leur caractère trop aléatoire quant aux chances de succès, les deux autres catégories peuvent être immobilisées de façon exceptionnelle, dès lors que deux conditions sont respectées: les projets 26 doivent être nettement individualisés et leur coût réparti dans le temps; chaque projet doit avoir de sérieuses chances de réussite technique et de rentabilité commerciale (PCG, p. II.38). Ces règles mettent en évidence l'importance de la notion d'" avantages économiques " qui enrichit et élargit le concept d'immobilisation. Voir Isabelle de Kerviler, Jean-Yves Obert, 
licences, les concessions et droits similaires ${ }^{14}$, les logiciels ${ }^{15}$, les marques, le fonds commercial, le droit au bail, l'investissement de création, et les autres droits et valeurs incorporels ${ }^{16}$. On retrouve la même liste dans la nomenclature budgétaire des collectivités territoriales de l'UEMOA ${ }^{17}$.

Le fonds commercial indiqué dans le «système comptable ouest africain" (SYSCOA) et inclus dans la totalité des dispositions de droit des affaires par l'OHADA, correspond à des éléments incorporels qui ne font pas l'objet d'une évaluation et d'une comptabilisation séparées au bilan et qui concourent au maintien ou au développement du potentiel d'activité de l'entreprise, de la clientèle, de l'achalandage, du droit au bail, du nom commercial et de l'enseigne. Ces éléments ne peuvent être inscrits à l'actif lorsqu'ils sont créés par l'entreprise elle-même. Seule l'acquisition permet de disposer d'un prix payé, vérifiable et objectif. Ce point est confirmé dans le cadre de la refonte 1999 du PCG. Le fonds commercial est défini dans l'Acte uniforme portant organisation

"Les investissements immatériels: solutions actuelles et perspectives", La Revue Banque, $\mathrm{n}^{\mathrm{0}}$ 503, 1990, p. 234-238.

14. Voir Jean-Luc Rossignol, op. cit., p. 10, «Les concessions, brevets, licences, marques, procédés, droits et valeurs similaires : il s'agit de dépenses que l'entreprise a effectuées pour l'obtention de l'avantage que constitue la protection accordée, sous certaines conditions, à l'inventeur, à l'auteur ou au bénéficiaire du droit d'exploitation d'une marque ou d'un brevet par exemple. Cet avantage a pu, selon les cas, être acquis auprès de tiers ou créé par l'entreprise. Il est l'objet ou non d'amortissement. L'amortissement est ainsi pratiqué sur les brevets 30 qui protègent l'invention sur 20 ans; il ne l'est pas sur les marques, en raison de la protection illimitée dont elles bénéficient et du fait qu'elles ont tendance à prendre de la valeur au fur et à mesure de leur utilisation.»

15. À propos des logiciels, la doctrine fait une distinction entre logiciels créés par l'entreprise et logiciels acquis, ainsi qu'entre la vocation interne de ces logiciels et leur vocation commerciale, voir Christine Afriat, L'investissement dans l'intelligence, Partis, PUF, «Que sais-je ?», 1992. Le logiciel acquis à titre onéreux ou créé par l'entreprise pour usage interne (ou comme moyen d'exploitation) constitue une immobilisation comptabilisée pour son coût d'acquisition ou de production. Voir Bull. CNCC $\mathrm{n}^{0}$ 114, juin 1999, p. 283-284: La commission des études comptables de la CNCC a indiqué que les dépenses de conception d'un site internet pouvaient être assimilées à la réalisation d'un logiciel.

16. Document de l'UEMOA, «Règles comptables spécifiques applicables aux intervenants agréés du marché financier régional de l'UEMOA», Annexe au Règlement 09/2006/CM/UEMOA, http://www.uemoa.int/sites/default/files/annexe/ annexes_09_2006_cm_uemoa.pdf.

17. Conseil des ministres de l'UEMOA, Nomenclature budgétaire des collectivités territoriales - UEMOA, http://www.uemoa.int/sites/default/files/bibliotheque/ nomenclature_budgetaire_collectivites_territoriales_uemoa.pdf. 
et harmonisation des comptabilités des entreprises ${ }^{18}$. Il faut cependant distinguer la notion comptable de «fonds commercial» de la notion juridique plus large de "fonds de commerce ${ }^{19}$ ". Même si la définition du fonds de commerce de l'Acte uniforme OHADA sur le commerce général indique les mêmes éléments que ceux contenus dans la définition comptable du fonds commercial, le fonds de commerce reste une notion imparfaitement cernée en droit, ce qui ne facilite pas son appréhension en comptabilité. Le fonds de commerce n'est défini, en fait, par aucun texte. En France, si la loi du 17 mars 1909 énumère les différents éléments qui peuvent servir à le constituer, il appartient au juge du fonds d'apprécier, d'après les éléments désignés par la convention, si ceux que les parties ont compris dans leurs opérations sont, ou non, des éléments essentiels sans lesquels le fonds de commerce ne saurait exister. Il est traditionnellement admis que le fonds de commerce est constitué par un ensemble d'éléments incorporels et corporels affectés à l'exploitation ou à l'exercice d'une activité commerciale. Ainsi, sont classés parmi les éléments incorporels: la clientèle et l'achalandage, le droit au bail, l'enseigne et le nom commercial, les brevets d'invention, les dessins et modèles industriels, les marques de fabrique ou de commerce, les licences, la propriété littéraire ou artistique, etc. Le matériel, l'outillage et les marchandises constituent les éléments corporels. La Cour de cassation française considère que la clientèle est un élément essentiel du fonds de commerce qui ne peut exister sans elle ${ }^{20}$.

Ces éléments listés dans les immobilisations incorporelles sont à mettre dans un fonds propre, selon la réglementation de Bâle, dont l'objectif est de prévenir des faillites bancaires à travers l'imposition d'un niveau minimal de fonds propres pour couvrir les risques ${ }^{21}$. L'existence des fonds propres en quantités suffisantes profite à la banque de différentes manières: avoir une position dominante sur le marché domestique pour préparer les futures acquisitions internationales ${ }^{22}$, se

18. Voir Annexes, chap. 2 (Contenu et fonctionnement des comptes), section 2, classe 2 (Comptes d'actifs immobilisés), compte 21 (Immobilisations corporelles), Subdivision 215, http://www.ohada.com/actes-uniformes/693/734/compte21-immobilisations-corporelles.html.

19. Jean Dupoux et Mohamed Helal, Le fonds de commerce: régimes juridique et fiscal, Paris, PUF, «Que sais-je ?», 1981.

20. «La clientèle est l'essence du fonds ». Cass. req. 15 février 1937, DP 1938. I. 13, note H. Rousseau.

21. Dominique Plihon, Jézabel Couppey-Soubeyran, Dhafer Saidane, Les banques acteurs de la globalisation financière, Paris, La Documentation française, 2006.

22. Medhi Nekhili et Catherine Karyotis, Stratégies bancaires internationales, Paris, Economica. 2008. 
protéger contre les prises de position hostiles ${ }^{23}$. Depuis janvier 2018, les nouvelles règles prudentielles applicables aux établissements de crédit de l'UEMOA sont entrées en vigueur, ces règles sont basées sur les Accords de Bâle II et III. Les responsables des banques se devaient de renforcer leurs capacités en matière de fonds propres. Il ne s'agit pas de revenir sur le bien-fondé ou non pour l'UEMOA d'adopter les règles de Bâle III, car d'une part, même si son système bancaire n'a pas eu à souffrir de la crise de 2008 , il n'en est pas à l'abri et, d'autre part, les règles de Bâle représentent des minimas internationaux et ne sont pas censées être appliquées uniformément. Le choix est laissé aux pays d'adopter des normes plus strictes ou de mettre leurs propres règlements en conformité avec les nouvelles normes dans un délai plus court ${ }^{24}$. Dans le même temps, certains sont sceptiques quant à la perspective de voir Bâle III éviter de futures crises bancaires ${ }^{25}$. Conjurer le sort d'une nouvelle crise dépend en grande partie des organes de régulation. La BECEAO, à ce propos, évalue la situation des immobilisations incorporelles et considère qu'elles ne doivent pas être intégrées dans la catégorie des autres actifs, car elles sont déjà déduites des fonds propres et par conséquent ne doivent pas être prises en compte dans les risques pondérés. D’où la question de savoir quel est le rôle dévolu aux immobilisations incorporelles dans les fonds propres. La question a son intérêt pour le droit comptable qui doit définir les critères de leur évaluation.

Afin d'y répondre, la première partie de cette analyse portera sur les enjeux stratégiques des immobilisations incorporelles pour les banques (1), la deuxième partie sera consacrée à leur traitement comptable par l'UEMOA (2).

23. Marie-Françoise Delaitte, «Le protectionnisme dans le capitalisme mondial », dans Mélanges en hommage à Jean-Claude Dischamps, Targoviste, Bibliotheca, 2009, p. $73-86$.

24. Éric Chouinard, Paulin Graydon, «La mise en œuvre de Bâle III : vers un secteur bancaire plus sûr ", Banque du Canada - Revue du système financier, 2014, p. 61-68 : "Ce qui importe en définitive, c'est que tous les pays resserrent suffisamment leurs normes de fonds propres et de liquidité. Le Programme d'évaluation de la concordance des réglementations avec Bâle III a d'ailleurs été mis sur pied par le Comité de Bâle pour assurer le respect intégral des normes internationales au moyen d'examens par les pairs."

25. Voir entretien avec Gilles Bonafi, "L'échec de Bâle III, un signal pour l'Afrique», Financial Afrik, avril 2014, https://www.financialafrik.com/2014/04/21/lechec-debale-iii-un-signal-pour-lafrique. 


\section{Les enjeux stratégiques des éléments incorporels}

Les éléments incorporels retenus dans la liste des immobilisations incorporelles rejoignent le fonds de commerce dont le contenu, établi par l'Acte uniforme du droit commercial général (AUDCG), prend en compte les éléments mobiliers, corporels et incorporels ${ }^{26}$. Tout comme le fonds de commerce constitue un investissement stratégique, les immobilisations incorporelles ont valeur d'enjeux stratégiques pour les banques, car elles sont des investissements. Cependant, leur évaluation peut s'avérer délicate, d'où l'importance de préciser la méthode comptable qui la réalise.

\subsection{Les éléments incorporels, investissements stratégiques}

Les pratiques comptables ne capitalisent pas les actifs immatériels issus des dépenses de recherche et développement, de publicité et d'informatique en tant que capital, parce que les entreprises ne fournissent généralement pas d'estimation distincte des coûts générés par les changements organisationnels ou les estimations des coûts d'ajustement de l'investissement et de la croissance ${ }^{27}$. Étant donné que les marchés financiers efficaces fourniront une estimation de la valeur boursière des sociétés cotées, y compris la valeur attribuée à tous les types d'actifs normalement invisibles dans les états comptables, une approche de l'évaluation des actifs incorporels d'une entreprise particulière a consisté à considérer sa valeur marchande et sa valeur comptable ${ }^{28}$. Cette approche a trois limites claires: premièrement, elle ne fait pas de distinction entre les actifs immatériels et incorporels ${ }^{29}$; deuxièmement, elle ne fait aucune distinction entre la valeur économique et le coût des actifs de

26. Art. 136-137 AUDCG.

27. Alfredo Martín-Oliver, Vicente Salas-Fumás, «How Do Intangible Assets Create Economic Value? An Application to Banks», Banco de España, Research Paper $\mathrm{n}^{\mathrm{o}} \mathrm{WP}-0730,2007$.

28. Robert E. Hall, «The stock market and capital accumulation», American Economic Review, vol. 91, $\mathrm{n}^{\mathrm{o}}$ 5, 2001, p. 1185-1202.

29. Les actifs incorporels peuvent être immatériels. Voir Éric Delesalle, «Dix ans d'application de la loi comptable », La documentation organique, 1994 : «de l'analyse du PCG et des pratiques, il faut constater que ce n'est pas le cadre actuel qui crée en tant que telles les difficultés de prise en considération des actifs incorporels immatériels, mais plutôt: - un manque de données précises, lié aux systèmes d'information des entreprises ; - un défaut de réflexion de la part des praticiens [...] ; - une certaine pratique empreinte de prudence ; - un défaut d'analyse des normalisateurs comptables sur les conditions et phases qui seraient envisageables pour permettre une comptabilisation de tels actifs. » 
l'entreprise ; et troisièmement, elle suppose, sans preuve, que les loyers provenant du pouvoir de marché de l'entreprise, le cas échéant, font tous partie de la valeur attribuée aux actifs incorporels ${ }^{30}$.

De manière générale, les éléments incorporels sont importants pour la compétitivité des entreprises, car les dépenses qu'ils occasionnent représentent des investissements stratégiques pour leur développement ${ }^{31}$. Ces dépenses ont été pendant longtemps considérées comme d'importance marginale ou résiduelle, parce qu'opposées à une richesse matérielle, obtenue avec des ressources matérielles ${ }^{32}$. Aujourd'hui, même cette richesse est reconnue et estimée comme étant très importante. La mesure la plus simple de la manière dont les actifs incorporels représentent l'économie provient des valeurs de marché des entreprises qui tirent la plus grande partie de leur valeur de ces actifs par rapport à l'ensemble du marché ${ }^{33}$. À ce propos, Baruch Lev, professeur Philip Bardes de comptabilité et de finances à la Stern School of Business de l'université de New York, pense qu'une façon de déterminer si l'estimation de la valeur des actifs incorporels est raisonnable est de comparer les valeurs de marché des sociétés avec les valeurs comptables (l'actif net) qui apparaissent dans leurs bilans, pour voir s'il y a un grand facteur non mesuré ${ }^{34}$. Ainsi, selon lui, les données pour les entreprises $\mathrm{S} \& \mathrm{P} 500^{35}$, qui représentent environ $75 \%$ du total des actifs de l'économie américaine, révèlent que depuis le milieu des années 1980, il y a eu une forte augmentation de la volatilité du ratio valeur/valeur du marché. À son apogée en mars 2000, le ratio de la valeur marchande à la valeur comptable était de 7,5. À la fin août 2002, il était de 4,2 et il peut encore baisser. Cependant, même si le ratio est tombé à 4 ou même à 3 , il serait plus important qu'il ne l'était dans les périodes précédentes, et il serait préférable de confirmer que la valeur des actifs incorporels est égale à la moitié et aux deux tiers des valeurs du marché des entreprises ${ }^{36}$.

30. Stephen Bond, Jason Cummins, "The stock market and investment in the new economy: Some tangible facts and intangible fictions », Brookings Papers on Economic Activity, 2000, p. 61-124.

31. Jean-Luc Rossignol, «La problématique comptable et fiscal des éléments incorporels (Première partie)», Petites Affiches, $\mathrm{n}^{\circ}$ 140, 2001, p. 4.

32. Ibid.

33. Aswath Damodaran, «Invisible Value? Valuing Companies with Intangible Assets », 2009, https://ssrn.com/abstract=1609799.

34. Baruch Lev, « Remarks on the Measurement, Valuation, and Reporting of Intangible Assets », Economic Policy Review, vol. 9, n 3, 2003.

35. Le Standard and Poor's 500.

36. Baruch Lev, op. cit. 
La rentabilité des éléments immatériels, contrairement à ce qui est couramment admis ${ }^{37}$, leur permet de prétendre au qualificatif d'investissement ${ }^{38}$. Selon Ahmed Bounfour ${ }^{39}$, une corrélation semble en effet établie entre l'accroissement des investissements immatériels et le développement d'avantages compétitifs sur le marché, témoignant ainsi de l'émergence d'un nouveau modèle de compétitivité caractérisé par une mutation des systèmes de production dans lesquels les activités immatérielles se substitueraient de plus en plus aux activités matérielles. Certains préfèrent leur appliquer le qualificatif de "quasi-investissement ", parce qu'ils n'ont ni la permanence, ni la fréquence, ni la reproductibilité des investissements physiques et que leurs effets bénéfiques restent incertains ${ }^{40}$. Ce débat terminologique illustre l'une des premières pistes de recherche dans le domaine de l'immatériel. En dépit de leur importance économique évidente, ces investissements constituent un sujet difficile et controversé qui a donné lieu à de nombreux travaux cherchant, par exemple, à en étudier l'impact sur les marchés financiers ou bien encore leur traitement en comptabilité ${ }^{41}$. Mais définir ces éléments relève déjà d'une problématique particulièrement délicate.

\subsection{Les difficultés d'évaluation des éléments incorporels}

Contrairement aux immobilisations corporelles qui sont bien délimitées comptablement ${ }^{42}$, les immobilisations incorporelles ${ }^{43}$ peuvent présenter des difficultés d'évaluation, notamment du fait que sur le plan comptable, elles font, en règle générale, l'objet d'un amortissement le plus souvent linéaire ${ }^{44}$, à l'inverse des autres éléments de l'actif qui, le cas échéant, font l'objet de provisions pour dépréciation. Cette remise

37. Derek Bosworth, "Returns to intellectual property in pharmaceuticals sector», Économie appliquée, vol. 52, $\mathrm{n}^{\circ}$ 2, 1999, p. 69-94.

38. Pamela Megna, Dennis C. Mueller, «Profit rates and intangible capital», Review of Economics and Statistics, vol. 73, $\mathrm{n}^{\mathrm{o}} 4$, 1991, p. 632-642.

39. Ahmed Bounfour, Le management des ressources immatérielles, maîtriser les nouveaux leviers de l'avantage compétitif, Paris, Dunod, 1998.

40. Rapport du Commissariat général du plan 1982, Paris, La Documentation française.

41. Ahmed Bounfour, op. cit

42. Elles comprennent les éléments suivants : terrains, agencement et aménagement des terrains, constructions, bâtiments, matériels et outillages, matériels de transport, matériels de bureau, les avances et acomptes.

43. Elles étaient autrefois qualifiées, par un processus d'élimination, de «toutes celles autres que les immobilisations corporelles». Voir Mémento pratique comptable, Paris, Francis Lefebvre, 1992, ${ }^{\circ} 1452$.

44. Alors que les autres éléments de l'actif, par exemple, font, le cas échéant, l'objet de provisions pour dépréciation. 
en cause de la pertinence du modèle comptable pour appréhender les éléments incorporels est souvent évoquée devant l'impossibilité de les évaluer de façon fiable, d'en saisir leur retour sur investissement et ainsi d'en garantir l'exactitude. Il est vrai que l'évaluation revêt une importance déterminante et que «l'interface symbolique-numérique, point d'entrée du modèle comptable, est l'une des sources de l'effet réducteur » qu'il présente ${ }^{45}$.

Pour être inscrit à l'actif, un bien doit être évalué en unités monétaires, ce qui peut exclure des éléments dont la valeur monétaire n'est pas immédiatement évidente (contrats d'exclusivité, obligation de ne pas faire, absence de pollution) ${ }^{46}$. Joseph-H. Vlaemminck ${ }^{47}$ exprime cette difficulté dans les termes suivants :

La démarche comptable doit donc analyser cet amas de faits. De cette observation et de cette analyse découlera la sélection. En effet, tous ces faits ne constituent pas des données comptables. La nature de la démarche comptable nous parait être telle que le premier critère est d'ordre quantitatif et non qualitatif. La comptabilité ne peut exprimer que des quantités. Sa première démarche sera, par conséquent, d'écarter tous les faits et tous les phénomènes irréductibles à la notion de quantité, tous ceux qui ne peuvent pas s'exprimer par un chiffre ou un nombre ${ }^{48}$.

Selon Alain Burlaud,

L'évaluation est l'acte nécessaire et préalable à tout enregistrement comptable. Mieux, l'évaluation est consubstantielle à la comptabilité puisque celle-ci ne sait traiter que des unités monétaires qui sont l'instrument par excellence de l'évaluation dans le domaine de l'économie et que celle-là ne peut bien souvent être réalisée sans le secours d'une comptabilité.

[...] sont ainsi délibérément écartés certains éléments non quantitatifs qui peuvent parfois constituer la substance même d'une firme: la capacité des dirigeants, la compétence de l'équipe de recherche et de développement, l'image de marque des produits, le climat social, les relations privilégiées avec l'État, etc. ${ }^{49}$

45. Jean-François Casta, Xavier Bry, «Synergy, financial assessment and fuzzy integrals », dans Proceedings of IVth Meeting of the International Society for Fuzzy Management and Economy (SIGEF), Santiago de Cuba, vol. 2, 1998, p. 17-42. Cahier de Recherche CEREG, $\mathrm{n}^{\circ}$ 2003-4, Université Paris Dauphine, 2003.

46. Jean-Guy Degos, Histoire de la comptabilité, Paris, PUF, «Que sais-je ?», 1998.

47. Joseph-H. Vlaemminck, Histoire et doctrines de la comptabilité, Bruxelles, Treurenberg, 1956.

48. Ibid.

49. Alain Burlaud, "Réflexions sur la gestion de la formation», colloque de la revue Politique et management public, Paris, 26 et 27 mars 1992. 
L'Acte uniforme du 24 mars 2000 portant organisation et harmonisation des comptabilités des entreprises de l'OHADA a prévu, en son article 35, la méthode d'évaluation et de détermination du résultat ${ }^{50}$. Parce que les méthodes d'évaluation prennent une place prépondérante dans la pertinence des états financiers, le système comptable OHADA y consacre de longs développements (art. 35 à 65). Différentes méthodes sont susceptibles d'être appliquées:

- le coût historique : coût d'acquisition ou de production de l'entreprise. Le principe de prudence conduit à lui substituer la valeur vénale à la date de clôture des comptes lorsqu'elle lui est inférieure.

- le coût historique indexé: valeur pour l'entreprise à la date de l'inventaire qui est constituée normalement, dans le cadre de la continuité de l'exploitation, par la valeur vénale de l'entreprise et, dans le cas contraire, par la valeur liquidative.

L'idée que la mesure de la valeur d'une entreprise ne peut être limitée à une simple somme d'actifs corporels nets est relativement récente. Elle émerge dans les démarches d'évaluation des entreprises vers 1925 et ne s'impose réellement qu'à partir de 1950. Glais et Sage ${ }^{51}$ citent la célèbre affaire Willot, au cours de laquelle le magistrat reconnut qu'il était essentiel de déterminer l'importance des éléments incorporels et d'en justifier la valeur, pour connaître celle des entreprises. De nombreuses méthodes d'évaluation existent pourtant, quels que soient leurs degrés de subjectivité et d'arbitraire, qui ne peuvent être éliminés d'aucune évaluation, de même que la perspective dans laquelle l'évaluateur se place (accords de licences, fusions-acquisitions...). Élisabeth Walliser fait remarquer à juste titre que «les investissements matériels sont eux aussi sujets au risque, en raison par exemple de l'obsolescence induite par l'évolution des techniques. Le risque qui pèse sur les profits futurs d'une entreprise concerne bien souvent, de manière indistincte, l'ensemble du potentiel productif, matériel et immatériel. Pour le $\mathrm{CNC}^{52}$, il n'est donc

50. Art. 35 : «La méthode d'évaluation des éléments inscrits en comptabilité est fondée sur la convention du coût historique et sur l'application des principes généraux de prudence et de continuité de l'exploitation. Cependant, il peut être procédé à la réévaluation des éléments dans des conditions fixées par les autorités compétentes, et dans le respect des dispositions des dispositions des articles 62 à 65 ci-après. »

51. Michel Glais et Eugène Sage, «Évaluation des actifs incorporels», Encyclopédie de Gestion, Yves Simon et Patrick Joffre (dir.), Paris, Economica, 1989, p. 1179-1198.

52. Note de l'auteur: Le Conseil national de la comptabilité (CNC), remplacé en 2009 par l'Autorité des normes comptables (ANC). 
guère raisonnable de suggérer que l'aléa puisse se focaliser de manière privilégiée sur les investissements immatériels ${ }^{53} \ldots$ »

Les deux approches retenues par le droit comptable OHADA semblent, en matière d'évaluation des actifs immatériels, pouvoir être retenues même en matière de marques, élément incorporel particulièrement fluctuant. La convention du coût historique est retenue en fonction de sa fiabilité, puisqu'elle conserve les flux pour leur montant d'origine, dans les valeurs d'entrée. Le système comptable OHADA applique ainsi le concept de maintien du capital de l'entreprise en francs ${ }^{54}$ courants, à l'exclusion des gains pouvant provenir de la détention de monnaies étrangères, d'instruments financiers, de compensations partielles en matière de titres ou de dettes et créances libellées en monnaies étrangères sous certaines conditions. Comme l'indiquent les dispositions de l'article 35, la méthode n'interdit pas la réévaluation des biens, mais celle-ci doit se faire dans des conditions fixées par les pouvoirs publics, il peut s'agir:

- d'une réévaluation légale dont la mise en ouvre et les modalités techniques sont strictement définies;

- d'une réévaluation libre à l'initiative de l'entreprise, dans les conditions prescrites.

En raison du principe de prudence, la valeur nette au bilan est la plus faible entre la valeur d'entrée et la valeur actuelle. La comptabilité doit donc pouvoir prendre en compte les éléments incorporels.

\section{La traduction comptable des éléments incorporels}

Le traitement comptable des immobilisations incorporelles diverge entre comptes sociaux et consolidés. Cette divergence de traitement s'explique, d'une part, par l'existence, en consolidation, de règles plus souples que celles qui régissent l'établissement des comptes sociaux, et d'autre part, par les limites inhérentes au modèle comptable traditionnel.

\subsection{Le contenu et le fonctionnement des comptes}

En pratique, la rubrique «immobilisations incorporelles » ne représente qu'une part négligeable du total des actifs des bilans des comptes sociaux des entreprises. Cette situation peut s'expliquer par le caractère

53. Élisabeth Walliser, «La prise en compte des marques à l'actif du bilan», Économie et comptabilité, $\mathrm{n}^{\circ} 196,1996$, p. 29-33.

54. Monnaie : franc CFA. 
confidentiel des éléments incorporels qui constituent l'un des paramètres fondamentaux de la stratégie concurrentielle des entreprises, et par le syndrome de la non-valeur qui peut les avoir affectées. Les explications classiques à cet état de fait reposent généralement sur deux aspects : l'un lié à la nature des dépenses en cause et plus particulièrement à la difficulté de leur évaluation, l'autre aux limites du modèle comptable et son adéquation pour retranscrire de telles dépenses, ce qui oblige les entreprises, contraintes de fournir de l'information financière en la matière, de recourir à d'autres documents.

Le droit comptable OHADA prévoit, dans son fonctionnement, que le compte 21, Immobilisations incorporelles, est débité de la valeur d'apport, d'acquisition ou de création par l'entreprise de l'immobilisation incorporelle: par le crédit du capital ${ }^{55}$, ou par le crédit du compte Associés et groupe $e^{56}$, ou par le crédit des comptes de tiers, ou par le crédit des comptes de trésorerie, ou par le crédit du compte Production immobilisée $e^{57}$.

Ainsi, la comptabilité qui n'a certes pas vocation à tout enregistrer (conception du «bilan intégral» $)^{58}$, ne serait pas à même de saisir la nature dynamique et immatérielle de la création de valeur. La comptabilité peinerait à appréhender au bilan ces dépenses invisibles, impalpables et parfois difficilement identifiables et individualisables, en raison de son caractère profondément marqué par une vision patrimoniale ${ }^{59}$ teintée de prudence ${ }^{60}$ et faute d'un cadre conceptuel lui permettant d'aborder l'ère informationnelle ${ }^{61}$. «Mode de traduction écrite de la réalité économique qui repose fondamentalement sur une analyse juridique de

55. Le compte 10.

56. Le compte 46 .

57. Le compte 72 .

58. Jean-Luc Rossignol, «La problématique comptable et fiscal des éléments incorporels (suite et fin)", Petites Affiches, $\mathrm{n}^{0}$ 141, 2001, p. 4.

59. Ahmed Bounfour (op. cit.). Il indique que « la notion de patrimoine étant entendue dans un sens davantage de patrimoine déjà constitué, c'est-à-dire passé, que de patrimoine en voie de constitution, c'est-à-dire à venir, en vertu de la règle de prudence imposée par la comptabilité générale ». Selon Boisselier (cité par Yassine Louzzani, Immatériel et performances des entreprises. Cas des entreprises industrielles en France sur la période 1994-1998, thèse de doctorat, 2004), le bilan représente le potentiel qu'a l'entreprise à générer de la valeur et non sa valeur ex ante: Patrick Boisselier, L'investissement immatériel, Bruxelles, De Boeck-Wesmael, 1993.

60. Selon Jean-Luc Rossignol (op. cit.), cette prudence interdit l'existence au bilan de certains éléments ou limite le montant pour lequel ils peuvent être inscrits.

61. Jean Cohen-Scali, «Information et relations, facteurs de développement ", Économie \& comptabilité, nº 199, juin 1997, p. 11. 
cette réalité ${ }^{62}$ ", la comptabilité connaîtrait des difficultés à décrire les mutations des entreprises dont les performances dépendent de nos jours et en grande partie de la qualité de leurs actifs immatériels, donc de leur capacité à entretenir et à accroître ce « $\operatorname{stock}^{63}$ ». Elle ne pourrait ainsi enregistrer exactement des «phénomènes économiques» qui sont de fait enregistrés parmi les charges, comme une dépense d'exploitation classique, suivant, en cela, la conception statique du bilan qui représente la doctrine dominante ${ }^{64}$.

La difficulté à évaluer les immobilisations incorporelles se ressent dans le commentaire que le droit comptable OHADA fait des différents éléments de l'immobilisation incorporelle ${ }^{65}$. En effet, s'agissant des frais de recherche et de développement, il est prévu que ne peuvent être immobilisées que les dépenses relatives à des projets qui ont de sérieuses chances de réalisation technique et de rentabilité commerciale en raison d'un marché potentiel. Aussi les projets concernés doivent-ils être nettement individualisés et leur coût distinctement établi pour être réparti dans le temps. Sont par conséquent exclus du champ des frais de recherche et de développement visés par le compte 211 les dépenses relatives à la recherche fondamentale, ainsi que les frais de lancement de la production.

Le compte 212, pour sa part, enregistre les dépenses engagées pour obtenir la protection accordée sous certaines conditions aux inventeurs, auteurs ou bénéficiaires du droit d'exploitation des brevets, modèles, dessins, procédés, propriétés littéraires et artistiques sous forme directe ou sous forme de licences ou de concessions. Le compte 213, Logiciels, enregistre les dépenses faites en vue d'acquérir le droit d'usage, d'adaptation, ou encore de reproduction d'un logiciel acquis, de même que le coût de production d'un logiciel créé ou développé pour les besoins internes de l'entreprise. Le logiciel lui-même est défini comme un ensemble de programmes, procédés, et règles assortis ou non de documentation, acquis ou créés par l'entreprise en vue du traitement automatique des données ${ }^{66}$.

62. Emmanuel du Pontavice, "Comptabilité et droit», Revue de droit comptable, $\mathrm{n}^{\mathrm{o}} 87-7$, mars 1987, p. 75-78.

63. Jean-Louis Médus, «L'incorporel et l'ingénierie financière», Droit et Patrimoine, mars 1996, p. 52-64.

64. Jacques Richard, Comptabilités et pratiques comptables, Dalloz, Paris, 1996.

65. http://www.ohada.com/actes-uniformes/693/734/compte-21-immobilisationscorporelles.html.

66. Ibid. 
Le compte 214, Marques, enregistre le coût d'acquisition des «marques» commerciales ou industrielles. Dans le cas où ces marques ne semblent pas avoir une valeur pérenne, elles sont à amortir. Le compte 215, Fonds commercial, est constitué par les éléments incorporels qui ne font pas l'objet d'une évaluation et d'une comptabilisation séparées au bilan et qui concourent au maintien ou au développement du potentiel d'activité de l'entreprise, de la clientèle, de l'achalandage, du droit au bail, du nom commercial et de l'enseigne. La clientèle et l'achalandage correspondent au potentiel de bénéfice représenté par l'existence d'une clientèle déterminée ou justifiée par l'emplacement de l'entreprise. Les éléments composant le fonds commercial ne bénéficient pas toujours d'une protection juridique leur donnant une valeur pérenne. Est seul inscrit à ce compte le fonds commercial acquis.

Le compte 216, Droit au bail, est constitué par le montant versé ou dû au locataire précédent en considération du transfert des droits résultant tant des conventions que de la législation sur la propriété commerciale. Le compte 217 , Investissements de création, se rapporte aux fabricants, producteurs, éditeurs et distributeurs de phonogrammes, aux entreprises de spectacle, aux établissements exerçant des activités culturelles et aux industries textiles (créateurs de mode). Sont donc portées au compte 217 les dépenses particulièrement élevées que les éditeurs engagent pour l'étude et la production de certains ouvrages et de certaines éditions (ouvrages de grandes collections, ouvrages d'art et encyclopédies), ainsi que les frais de collection exposés dans l'industrie textile.

En ce qui concerne les «immobilisations incorporelles en cours», le compte 219 enregistre le coût de production des brevets, investissements de création et logiciels élaborés par l'entreprise elle-même, dont les éléments transitent pour la plupart par le compte 211, Frais de recherche et de développement.

\subsection{La cession et l'amortissement des immobilisations}

En tout état de cause, même en cas d'absence ou d'insuffisance de bénéfices, l'entreprise procède aux amortissements et aux provisions nécessaires pour que le bilan donne une image fidèle du patrimoine, de la situation financière et du résultat de l'exercice. Le modèle comptable montre ainsi ses limites. En effet, la problématique comptable des éléments incorporels pose la question de la pertinence du système comptable dans son ensemble, dans la mesure où les documents comptables ne mettraient pas en évidence tous les faits pouvant avoir une influence 
sur le jugement que les destinataires de l'information peuvent porter sur le patrimoine, la situation financière et les résultats de l'entreprise. Pour Michel Prada, «le jeu combiné de l'amplification du cycle économique, d'une activité faisant une place toujours plus importante à l'immatériel et de la globalisation des marchés, a provoqué une certaine crise de crédibilité des dispositifs d'information comptable ${ }^{67} »$. La capacité de la comptabilité à appréhender, dans un environnement instable $e^{68}$, la réalité économique et financière, du fait de l'existence de postulats et conventions qui structurent la représentation de l'entreprise donnée par les états financiers ${ }^{69}$, est de fait fortement mise en défaut. Une telle problématique remet en cause l'exhaustivité du paradigme de l'entreprise patrimoniale comme représentation des structures actuelles de l'organisation ${ }^{70}$.

Le droit comptable OHADA crédite le compte 21 en cas de cession, disparition, destruction ou mise au rebut, d'abord par le débit $\mathrm{du}$ compte Valeurs comptables des cessions d'immobilisations ${ }^{71}$ ou du compte Valeurs comptables des cessions courantes d'immobilisations ${ }^{72}$; ensuite, et/ou par le débit du compte Amortissements des immobilisations incorporelles ${ }^{73}$ (pour solde de ce compte). L'amortissement est la constatation comptable obligatoire de l'amoindrissement de la valeur d'une immobilisation qui se déprécie de façon certaine et irréversible avec le temps, l'usage, ou en raison du changement des techniques, de l'évolution des marchés, de toutes autres techniques, ou de toute autre cause. Du fait des difficultés de mesure de cet amoindrissement, l'amortissement procède d'une répartition programmée selon un plan d'amortissement du coût du bien sur sa durée probable d'utilisation. Toute modification significative des conditions d'utilisation du bien justifie la révision du plan d'amortissement en cours d'exécution.

67. Michel Prada, Intervention au $\mathrm{XV}^{\mathrm{e}}$ Congrès mondial de la comptabilité, le 28 octobre 1997, reprise dans Bull. COB, $\mathrm{n}^{\circ} 317$, octobre 1997, p. 1 et suiv.

68. Brigitte Raybaud-Turillo, Robert Teller, «Comptabilité créative», dans Encyclopédie de gestion, op. cit., t. 1, p. 508-527.

69. Christian Hoarau, « Un bilan pour un vingtième anniversaire », dans « Les vingt ans de l'AFC », Comptabilité, contrôle, audit, 1999, p. 5-12.

70. Brigitte Raybaud-Turillo, «Droit comptable et droit économique : une approche renouvelée de la patrimonialité », Comptabilité, contrôle, audit, vol. 1, nº 1, mars 1995 , p. 25-44.

71. Le compte 81.

72. Le compte 654 .

73. Le compte 281 . 
Il ne faut pas confondre l'amortissement des immobilisations avec l'amortissement du capital, ni avec l'amortissement d'un emprunt, lequel signifie remboursement. De même, l'amortissement dérogatoire ne correspond pas à un amoindrissement de la valeur d'une immobilisation. Il s'agit d'une provision réglementée. Ainsi donc, les éléments du compte 212, Brevets, licences, concessions et droits similaires, sont amortissables sur leur durée de vie économique au maximum égale à la durée de la protection juridique.

Le compte 28 dans le droit comptable OHADA pose le principe général de l'obligation d'amortir les immobilisations corporelles et incorporelles, et ceci doit se faire même en cas d'absence ou d'insuffisance de bénéfice. Du reste, l'absence d'amortissement est constitutive du délit de présentation de faux bilan, car il n'est pas donné une image fidèle de la «richesse» de l'entreprise ${ }^{74}$. Les marques échappent toutefois à l'obligation d'amortissement en raison du fait qu'elles peuvent être renouvelées indéfiniment, contrairement aux autres éléments constitutifs du droit de la propriété intellectuelle que sont les brevets, les modèles et le logiciel.

L'on voit donc que la matière des immobilisations incorporelles est complexe. Elle devient alors difficile à gérer lorsque l'on passe au niveau de la garantie de l'actif et du passif.

L'enregistrement en charge des éléments incorporels, bien que simple et vérifiable, peut apparaître non pertinent dès lors que ces derniers peuvent avoir un impact important et durable sur la rentabilité de l'entreprise. En outre, il est impossible de les faire apparaitre au bilan d'exercices ultérieurs, lorsque leurs effets sur le résultat, attestant de leur valeur en tant qu'éléments d'actif, auront vu le jour. $\mathrm{La} \mathrm{COB}^{75}$ a indiqué, dans son rapport pour 1989, que «le développement du secteur tertiaire, où les actifs incorporels ont souvent plus d'importance que les autres [...], ont rendu les conséquences de la méconnaissance des éléments incorporels de plus en plus dommageables pour la qualité des documents comptables $^{76}$ ».

74. Jean Calvo, Alain Couret, "Garantie d'actif et de passif (aspects pratiques) - Les immobilisations incorporelles ", Petites Affiches, $\mathrm{n}^{\circ}$ 8, 1995, p. 9.

75. La Commission des opérations de bourse.

76. Bull. $\mathrm{COB}, \mathrm{n}^{\circ} 223$ à 231 . 


\section{Conclusion}

Au-delà du cas des immobilisations incorporelles et de leur prise en compte dans les fonds propres des institutions bancaires ouestafricaines, se profile à l'horizon le rôle de plus en plus accru que vont devoir jouer aussi bien l'organe de régulation du système bancaire qu'est la BECEAO que les états actionnaires. L'État a le devoir de contrôler les banques qui ne sont pas des entreprises comme les autres. L'UEMOA n'a pas choisi le contrôle de la banque par l'État en vue d'influencer sa gestion et mener une stratégie patrimoniale plus orientée vers la profitabilité à moyen terme. Certains auteurs ${ }^{77}$ préconisent en effet cette intervention publique afin de faciliter l'octroi de crédits immédiats à l'économie, ce qui serait plus utile pour l'ensemble des acteurs économiques que de conforter actionnaires et créditeurs ${ }^{78}$.

L'UEMOA a plutôt choisi une réforme globale de ses instruments de gestion de la stabilité financière. Cela implique une réforme de la supervision microprudentielle, dont l'objectif est la mise en conformité avec les principes fondamentaux du Comité de Bâle. Une implication majeure de cette mise en conformité est l'adoption de la supervision sur base consolidée des groupes bancaires. Le deuxième volet de cette réforme est la mise en place d'instruments complémentaires qui agissent au niveau de l'ensemble du système financier: fonds de garantie des dépôts, fonds de stabilité financière, identification des établissements bancaires d'importance systémique, mécanisme de résolution des crises et adoption d'une politique macroprudentielle ${ }^{79}$.

Il ne faut pas perdre de vue que les nouvelles normes qui sont entrées en vigueur depuis janvier 2018, pourraient également avoir des coûts économiques après leur mise en œuvre complète. Entre autres, les banques de la zone UEMOA peuvent chercher à compenser les coûts associés au resserrement des exigences de fonds propres et de liquidité en abaissant leurs taux de rémunération des dépôts, en

77. Jean-Paul Fitoussi, Joseph Stiglitz, «Le "Gn fantôme". Les voies pour sortir de la crise en renforçant la solidarité mondiale», Revue de l'OFCE, n 110, 2009, p. $472-482$.

78. Dominique Plihon, Le nouveau capitalisme, Paris, La Découverte, 2009. L'auteur affirme que la nationalisation devrait permettre un contrôle social, c'est-à-dire une nouvelle gouvernance bancaire à laquelle doivent participer l'ensemble des parties prenantes : État, salariés, usagers.

79. Samuel Guerineau, Michaël Goujon, Relwende Sawadogo, «Les politiques macroprudentielles dans l'UEMOA ", Rapport Fondation pour les études et recherches sur le développement international (Ferdi), 2016. 
majorant leurs taux d'intérêt sur prêt, ou en augmentant leurs frais de service ${ }^{80}$. Des préoccupations ont aussi été exprimées au sujet des répercussions possibles de Bâle III sur le fonctionnement du marché financier ${ }^{81}$. Une évaluation des résultats de cette réforme du système bancaire de l'UEMOA dans quelques années dira si le renforcement des règles prudentielles a produit les effets escomptés ou non. D'autant plus que dans le système bancaire, la comptabilité ne tient pas toujours compte de ce qu'on appelle les «immobilisations incorporelles non enregistrées ${ }^{82}$ ». En effet, si la comptabilité mesure parfaitement les aspects économiques sous-jacents d'une entreprise, il n'existe aucun bénéfice anormal. Cependant, la comptabilité est biaisée de manière prévisible et l'utilisation de la comptabilité en coût historique ne reflète pas certaines transactions ou certains soldes. Par conséquent, les loyers économiques, les opportunités de croissance et d'autres facteurs ne sont pas pleinement pris en compte dans le système de comptabilisation des coûts historiques et des résultats anormaux résultent de ces biais comptables $^{83}$.

Une association significative et positive entre le niveau des actifs incorporels non comptabilisés des banques et les résultats anormaux a été démontrée par certaines études ${ }^{84}$. Il a été trouvé une association d'actifs incorporels aux résultats anormaux plus forte pour les banques présentant des proportions plus élevées d'actifs incorporels de dépôt. Mais les résultats ne semblent pas généralisables sur la base d'une analyse des actifs incorporels de recherche et développement non enregistrés dans les entreprises manufacturières.

80. Ces effets s'atténueront, cependant, au fil du temps par la réduction des coûts de financement des banques. Cette réduction devrait intervenir à mesure que le risque associé au système bancaire diminuera.

81. Éric Chouinard, Graydon Paulin, «La mise en œuvre de Bâle III : vers un secteur bancaire plus sûr ", Revue du système financier, juin 2014, p. 61-68.

82. Ces actifs incorporels pas toujours enregistrés représentent les flux de trésorerie futurs prévus (économies), dérivés des relations clients existantes liées aux dépôts de base, au service des prêts hypothécaires, aux cartes de crédit et aux opérations fiduciaires. Le plus important de ces actifs incorporels, l'immatériel des dépôts de base, quantifie l'avantage favorable du financement des dépôts d'une banque. Les trois autres actifs incorporels des banques présentent des caractéristiques similaires, reflétant des flux de trésorerie supplémentaires provenant des relations clients existantes pour le service des prêts hypothécaires, les cartes de crédit et les opérations fiduciaires.

83. Mark J. Kohlbeck, Terry D. Warfield, « The Role of Unrecorded Intangible Assets in Residual Income Valuation: The Case of Banks », UW-Madison AIS Working Paper, $\mathrm{n}^{\mathrm{o}} \mathrm{KW} 2002$, janvier 2002.

84. Ibid. 
Ces résultats sont importants, car ils identifient les informations pouvant être utilisées pour améliorer la prise en compte des immobilisations incorporelles. Il y a d'ailleurs aujourd'hui une évolution de la pertinence en valeur des informations comptables ${ }^{85}$, qui se traduit par la prise en compte dans le droit comptable de l'OHADA, en ce qui concerne les actifs incorporels, entre autres des frais de recherche et développement, des actifs incorporels comptabilisés et des frais de publicité. En fournissant des éléments de preuve sur les facteurs contribuant à des gains anormaux, les banques de la zone UEMOA devraient être en mesure de prédire de futurs gains anormaux, qui constituent un élément clé de la valorisation des immobilisations incorporelles. Heureusement, les investisseurs et les analystes financiers s'intéressent de plus en plus aux actifs incorporels et, en matière d'investissement, les immobilisations incorporelles ont une influence sur la détermination et la prévision de la valeur de l'entreprise dans les secteurs où des mesures pertinentes et fiables de ces immobilisations incorporelles sont disponibles.

Docteur en droit Faculté de Droit, université de Lomé

85. Mary E. Barth, Ken Li, Charles G. McClure, «Evolution in Value Relevance of Accounting Information", Stanford University Graduate School of Business Research Paper, ${ }^{\circ}$ 17-24, 2018. 